\section{Validación de escalas de seguridad alimentaria y de apoyo social en una población afro-colombiana: aplicación en el estudio de prevalencia del estado nutricional en niños de 6 a 18 meses}

\author{
Validation of food security and social support \\ scales in an Afro-Colombian community: \\ application on a prevalence study of nutritional \\ status in children aged 6 to 18 months
}

Beatriz Eugenia Alvarado 1 María Victoria Zunzunegui ${ }^{2}$ Helene Delisle ${ }^{3}$

\footnotetext{
1 Unidad de Epidemiología Clínica, Universidad del Cauca, Popayán, Colombia.

2 Département de Médecine Sociale et Préventive, Université de Montréal, Montréal, Canada.

3 Département de Nutrition, Université de Montréal, Montréal, Canada.

Correspondencia B. E. Alvarado Unidad de Epidemiología Clínica, Universidad del Cauca. 5200 Anthony Drive, Apt 713, Detroit,

MI 48202, U.S.A.

be.alvarado.llano@umontreal.ca bealva@unicauca.edu.co
}

\begin{abstract}
We conducted a cross-sectional study on 193 mothers of children 6 to 18 months of age in an African-Colombian community, with the objectives: (1) to adapt and validate the Community Childhood Hunger Identification Project scale, the DUKE-UNC-11 social support scale, and the Quebec Longitudinal Study of Child Development (QLSCD) partner support scale, and (2) to identify any existent relationship between nutritional status in infancy and both food insecurity and social support. We determined construct validity using factor analysis and theoretical models-based non-parametric correlations. Lengthfor-age and weight-for-length Z-results were calculated. Factor analyses reduced the hunger scale to one factor, the DUKE-UNC-11 scale to two factors, and the QLSCD scale to one factor. The Cronbach's alpha test ranged between 0.70 and 0.90. Both food insecurity and social support scales were correlated with mother's social conditions, and social support was positively associated with social networks and mother's self-perceived health status. Food insecurity, emotionalsocial support, and partner's negative support were associated with lower height-to-age and therefore a higher ratio of chronic malnutrition. The study supports the appropriateness of the instruments to measure the expressed concepts.
\end{abstract}

Social Support; Nutritional Status; Child Welfare

\section{Introducción}

Los recursos para el cuidado (resources for care) son un elemento importante para que las prácticas de cuidado infantil sean efectivas 1 . Investigaciones realizadas en Bangladesh, Nigeria, Indonesia, Nicaragua y México 2,3 muestran que los recursos materiales (medidos por las condiciones de la vivienda y el presupuesto para la comida) afectan las prácticas de alimentación, de higiene y de atención en salud; y que los recursos sociales (medidos como el nivel educativo de la madre y el apoyo social) afectan las prácticas de afecto y atención del niño. Estudios recientes muestran, también, el efecto directo de los recursos para el cuidado sobre el estado nutricional infantil 4,5,6. Sin embargo, en América Latina no se tienen datos recientes sobre el papel que juegan la seguridad alimentaria y el apoyo social en el mantenimiento del estado nutricional infantil. La revisión bibliográfica sugiere que la falta de instrumentos adaptados a nuestras poblaciones podría estar explicando la falta de estudios 1,7 .

En miras a responder a esta inquietud en este informe se describirá la adaptación y la validez de tres instrumentos para medir: la seguridad alimentaria, el apoyo social general y el apoyo social del padre del niño. Se utilizaron en nuestra población la encuesta de hambre realizada por el Community Childhood Hunger Identification Project (CCHIP) 8 (Tabla 1), el 
cuestionario de apoyo social de Duke-UNC-11 9, y la encuesta de apoyo social del compañero del Québec Longitudinal Study of Child Development (QLSCD) 10 (Tabla 2). La escala de seguridad alimentaria fue adaptada en una zona urbana de bajos recursos económicos en Venezuela y mostró validez de constructo con un modelo teórico que incluye las condiciones sociales de las mujeres encuestadas 8 . La escala de apoyo social de Duke-UNC-11 es una escala sencilla y breve, validada en español 11, que comprende tres dimensiones: afectiva, instrumental y confidencial. Una calificación baja en la escala de apoyo de Duke-UNC-11 ha sido asociada con maltrato en niños 12 . Finalmente, la escala del QLSCD reúne en 5 preguntas las dimensiones de afecto, apoyo instrumental y confidencialidad del padre del niño. La escala ha sido desarrollada para identificar los determinantes sociales del crecimiento en una muestra de 2.223 niños, de varios orígenes étnicos en Québec 10. Las escalas de seguridad alimentaria y apoyo social antes mencionadas no han sido utilizadas para el estudio de la relación de estos recursos de cuidado sobre el estado nutricional infantil. En este informe se presentan los análisis preliminares que dan cuenta de esas relaciones en un estudio de prevalencia de desnutrición en una población afro-colombiana. Los instrumentos que se presentan en este estudio pueden ser adaptados y replicados en otras poblaciones latinoamericanas.

\section{Métodos}

\section{Diseño}

Los datos presentados forman parte de un estudio de prevalencia realizado en el área urbana del Municipio de Guapi, Costa Pacífica Colombiana, que tiene como objetivo identificar los determinantes sociales y biológicos del estado nutricional en niños de 6 a 18 meses. La población del municipio se calcula en 30.000 habitantes, 18.000 de ellos habitando en el casco urbano. El 90,0\% de la población del municipio está constituida por afro-colombianos con un bajo porcentaje de población mestiza y de comunidades indígenas. La zona del estudio se caracteriza por acceso inadecuado a servicios básicos y condiciones de vivienda precarias 13 . La economía es de sustento, siendo las principales ocupaciones: la pesca artesanal, la tala de madera y la agricultura de pequeña es- cala. Las ocupaciones de mayor estatus dentro de la población están formadas por los educadores, administradores y trabajadores de la salud. El acceso a la cabecera urbana se hace generalmente por vía marítima o aérea, dificultando el acceso de alimentos y otros productos no disponibles localmente. En la región se cultivan tubérculos como el plátano y la papachina, el arroz, la caña y algunos frutales como el coco, el naidi, la guaba, el chontaduro y el mamey. Algunas casas tienen cultivos para consumo familiar, al igual que criaderos de animales como pollos, gallinas y cerdos. El perfil de morbilidad infantil en esta región muestra que las enfermedades diarreicas agudas, las infecciones respiratorias, la malaria y otras enfermedades parasitarias son las cinco principales causas de consulta y hospitalización. A nivel local, no existen reportes de prevalencia de malnutrición crónica, pero la Encuesta Nacional de Demografía y Salud del año 200014 estima que para la región pacífica la prevalencia es del orden del 9,8\%.

\section{Participantes}

El diseño del estudio fue evaluado y aprobado por los Comités de Ética de la Universidad de Montreal y de la Organización Panamericana de la Salud. Inicialmente, se realizó un censo de niños nacidos entre Diciembre del 2001 y Diciembre del 2002 en el total de barrios que constituyen la cabecera de Guapi. Todas las madres sin excepción fueron invitadas a participar. Las madres censadas recibieron un formato de participación que informaba los objetivos, beneficios, riesgos y confidencialidad del estudio. La información se recogió entre julio y octubre de 2002. Diez madres (10) no se encontraron en tres visitas consecutivas, 4 no aceptaron participar en el estudio y 27 habían cambiado de lugar de residencia. Un total de 202 madres aceptaron participar. Ciento noventa y tres madres (193) proveyeron información completa para ser utilizada en nuestros análisis.

\section{Recolección de la información}

El cuestionario total constaba de 11 secciones: características sociodemográficas de la madre y padre del niño, las condiciones de la vivienda, la estructura familiar, la red social, la salud materna, el estado de salud del niño, las prácticas de alimentación del niño, la seguridad alimentaria, la frecuencia de consumo de alimen- 
Cuestionario de inseguridad alimentaria (Community Childhood Hunger Identification Project).

En los últimos 6 meses ha faltado dinero en el hogar para comprar alimentos

Nunca

A veces

Casi siempre

Siempre

En los últimos 6 meses ustedes han disminuido el número usual de comidas por falta de dinero en el hogar

En los últimos 6 meses ustedes han disminuido el número de comidas de algún ADULTO por falta de dinero en el hogar

En los últimos 6 meses algún ADULTO come menos en la comida principal porque los alimentos no alcanzan

En los últimos 6 mese, algún ADULTO en su casa se queja de hambre por falta de alimentos

En los últimos 6 meses algún ADULTO se acuesta con hambre porque no alcanza la comida

En los ultimos 6 meses algún miembro de su familia come menos de lo que desea por falta de dinero en el hogar

En los últimos 6 meses ustedes han disminuido el numero de comidas de algún NIÑO por falta de dinero en el hogar

En los últimos 6 meses algún NIÑO come menos en la comida principal porque los alimentos no alcanzan

En los últimos 6 meses algún NIÑO en su casa se queja de hambre por falta de alimentos

En los últimos 6 meses se compran menos alimentos indispensables para los NIÑOS por falta de dinero (leche, pescado, huevos, frutas, legumbres...)

En los últimos 6 meses algún NIÑO se acuesta con hambre porque no alcanza la comida

\section{Nunca}

A veces

Casi siempre

Siempre

Nunca

$A$ veces

Casi siempre

Siempre

Nunca

A veces

Casi siempre

Siempre

Nunca

$A$ veces

Casi siempre

Siempre

Nunca

A veces

Casi siempre

Siempre

Nunca

$A$ veces

Casi siempre

Siempre

Nunca

$A$ veces

Casi siempre

Siempre

Nunca

$A$ veces

Casi siempre

Siempre

Nunca

A veces

Casi siempre

Siempre

Nunca

$A$ veces

Casi siempre

Siempre

Nunca

A veces

Casi siempre

Siempre 
Escala de apoyo social del compañero (Québec Longitudinal Study of Child Development).

\begin{tabular}{|c|c|c|c|c|c|c|c|}
\hline & 1 & 2 & 3 & 4 & 5 & 6 & 7 \\
\hline \multicolumn{8}{|l|}{ 1. Su compañero la ayuda con su hijo } \\
\hline \multicolumn{8}{|c|}{ 2. Su compañero la ayuda en las cosas de la casa } \\
\hline \multicolumn{8}{|c|}{ 3. Su compañero la ayuda cuando está cansada } \\
\hline \multicolumn{8}{|c|}{ 4. Su compañero la ayuda cuando está muy triste } \\
\hline 5. En general su compañero la ayuda & & & & & & & \\
\hline
\end{tabular}

tos y el apoyo social. La información se recogió en el domicilio de las madres, por cinco entrevistadoras previamente entrenadas. Las primeras entrevistas fueron asistidas por la investigadora principal y los datos confirmados en una segunda visita. Las encuestadoras fueron todas mujeres de la región, cuya escolaridad mínima fue la secundaria.

\section{Mediciones}

\section{- Seguridad alimentaria}

En 12 preguntas la escala de índice de hambre (8) valora la percepción de la madre con respecto a la falta de consumo de alimentos, modificaciones de la dieta por falta de dinero y de alimentos, o experiencias de hambre en niños y adultos durante los últimos 6 meses (Tabla 1). Las respuestas fueron consignadas como: nunca (0), a veces (1), casi siempre (2) y siempre (3). La prueba piloto de la encuesta fue realizada entre 20 madres con niños menores de 2 años en la región de estudio. Las frases incluidas en el cuestionario final fueron idénticas a las que fueron formuladas por Lorenzana \& Sanjur 8. La secuencia de las preguntas fue ajustada dejando los ítems de los adultos al inicio y de los niños al final de la encuesta (Tabla 1).

\section{- Apoyo social}

El cuestionario de Duke-UNC-11 evalúa a través de 11 preguntas el apoyo social percibido 11: confidencial (posibilidad de contar con personas para comunicarse), afectivo (demostraciones de amor, cariño y empatía) e instrumental (ayuda o asistencia en necesidades tangibles). En la prueba piloto, la escala visual de caras mostró mayor grado de comprensión que la escala de Likert, usada en el estudio de validación. Los ítems fueron transformados en frases de manera que pudieran ser aplicados a manera de entrevista, debido a la baja escolaridad de la población de estudio. El cuestionario fue aplicado por una de las investigadoras (B. E. A.) con el objeto de aumentar la confidencialidad de las personas entrevistadas. Las categorías de respuesta fueron codificadas de -3 a 3 , que toma valores positivos a mayor grado de apoyo. El apoyo del compañero o esposo se evaluó mediante la escala de 5 ítems utilizada en el QLSCD 10. La escala no ha sido evaluada en poblaciones de habla española. El cuestionario fue traducido al español por una persona bilingüe en francés-español. La medición de las categorías de respuesta se realizó con la escala visual de caras y los códigos asignados fueron similares a los que se utilizaron para la escala Duke-UNC-11. La escala mostró en la prueba piloto sencillez en la comprensión y rapidez de la respuesta.

\section{- Estado nutricional del niño}

La talla fue medida en posición decúbito. Un tallímetro de madera con apoyo en la cabeza y una base móvil que se frena con el contacto de los pies fue utilizado en todos los niños. La medición de la talla fue realizada por un investigador (B. E. A.) y por dos auxiliares de enfermería previamente entrenadas (error técnico menor de $4 \mathrm{~mm}$ ). El promedio de cuatro (4) medidas fue tomado para calcular los índices antropométricos. El peso del niño se realizó con una balanza suspendida (Detecto, $25 \mathrm{~kg} / 500 \mathrm{~g}$ ). La misma balanza fue utilizada en todos los niños y calibrada antes cada toma. El niño fue pesado completamente desnudo en dos oportunidades. El promedio de las dos medidas se utilizó para los análisis. La talla y el peso del niño fueron transformados a $\mathrm{Z}$ resultados usando Epi Info 6.04, según la población de referencia aceptada por la Organización Mundial de la Salud. Los valores Z para talla-para-edad y pesopara-talla fueron calculados. Los datos presentaron una distribución normal y fueron tratados de manera continua en nuestros análisis. 


\section{- Variables predictoras}

De acuerdo a modelos teóricos 8, la escala de seguridad alimentaria debe relacionarse con el nivel de escolaridad de la madre, las condiciones socioeconómicas, el tamaño y la composición del hogar, el trabajo de la mujer y el ingreso familiar. Para la medición del nivel de escolaridad se consideró el número de años de estudio, y para las condiciones socio-económicas el número de pertenencias en el hogar - nevera, electricidad, teléfono, estufa y radio. Para medir el tamaño y composición del hogar se determinó el índice de dependencia (el número de personas en la vivienda menores de 15 años/mayores de 15 años). Los ingresos de la madre se categorizaron de acuerdo a si estaban por debajo o arriba del salario mínimo mensual para Colombia en el año 2003 (US\$ 110). Igualmente, el apoyo social se estructura por condiciones socioeconómicas y el nivel de escolaridad según La Teoría de las Redes Sociales 15. Siguiendo está teoría, las redes sociales (el numero de familiares/amigos, el numero de contacto visuales con amigos/familiares, y el grado de integración social) y el estado civil, proveen oportunidades para el apoyo social. El apoyo social se relaciona con los estados de salud, y un buen indicador es la salud percibida 15 . Las redes sociales fueron definidas a partir de dos preguntas: ¿Cuántos amigos/tíos/primos/ hermanos tiene?; ¿A cuántos de sus amigos/ tíos/primos/hermanos ve al menos una vez al mes? La integración social se definió a partir de la asistencia mensual a la (a) iglesia, (b) reuniones sociales, (c) fiestas, (d) juegos, (e) reuniones de barrio y (f) grupos de madres, asignando valor de 0 a la falta de asistencia. Las variables de redes sociales, estado civil y salud percibida fueron categorizadas de acuerdo a nuestros análisis descriptivos como se muestra en la Tabla 3.

\section{Análisis}

Los datos de este estudio fueron analizados utilizando el programa SPSS 10.0. Se exploraron las dimensiones de la escala de hambre y de apoyo social a través de los análisis factoriales. Los métodos de extracción utilizados fueron sucesivamente: componentes principales, ejes principales y máxima verosimilitud. La consistencia interna se midió con el alfa de Cronbach. Para medir la validez de constructo se observo la distribución de las variables seguridad y apoyo social, según las variables predictoras y se calcularon los coeficientes de correlación de Spearman. Finalmente, se calcula- ron los promedios de $\mathrm{Z}$ resultado de talla-paraedad y peso-para-talla, a través de las categorías de seguridad y apoyo social utilizando los procedimientos GLM (General Linear Model tipo III). En los análisis se probó el efecto de las variables de edad y sexo del niño, y la talla y peso maternos, como variables de confusión y predictoras de potencial genético, respectivamente. Mediante prueba de diferencia de proporciones, se compararon los porcentajes de desnutrición aguda $(<-2$ DE en peso-para-talla) y crónica ( $<-2$ DE en talla-para-edad) entre las categorías de seguridad alimentaria y apoyo social.

\section{Resultados}

\section{Seguridad alimentaria}

Los resultados del análisis de factores para la escala de inseguridad alimentaria mostraron la presencia de un factor (Tabla 4) con alta consistencia interna. El factor único explicó el 67,1\% de la varianza total, la saturación de cada ítem en el factor osciló entre 0,54 y 0,89. La correlación promedio entre ítem fue de 0,62 (rango: 0,33-0,83). Representados en los promedios más altos (Tabla 4), la inseguridad alimentaria se caracteriza principalmente por falta de dinero para la compra de alimentos, disminución en el número de comidas o disminución en la compra de alimentos indispensables para los niños. Los promedios más altos se describen para la inseguridad en adultos que para los niños (Tabla 4). Las categorías de respuesta (0 a 3) fueron sumadas a partir de sus valores iniciales para crear el índice de inseguridad alimentaria, que se distribuyó con un promedio de 9,31. El resultado de inseguridad alimentaria fue categorizado siguiendo los resultados de Lorenzana \& Sanjur 8: (0) seguro, (1-12) levemente inseguro, (13-24) moderadamente inseguro y (más de 25) severamente inseguro. La seguridad alimentaria fue infrecuente para la población de estudio (17,0\%). El porcentaje de personas viviendo en hogares en las categorías mediana, moderada y severamente inseguras fueron $61,6 \%$ (119), $17,6 \%$ (34) y $3,6 \%$ (7), respectivamente.

\section{Apoyo social}

En la escala de apoyo social los ítems 1, 2, 3 y 9 fueron excluidos por comunidades bajas (menor 0,30 ). La solución de ejes principales con rotación oblimin fue retenida como modelo final (Tabla 5). El primer factor reúne los ítems 
Distribución de inseguridad alimentaria y apoyo social por niveles de las variables predictoras. Correlaciones parciales no paramétricas

\begin{tabular}{|c|c|c|c|c|c|c|c|c|c|c|c|c|}
\hline \multirow[t]{2}{*}{ Variables predictoras } & \multicolumn{3}{|c|}{$\begin{array}{l}\text { Inseguridad } \\
\text { alimentaria }\end{array}$} & \multicolumn{3}{|c|}{$\begin{array}{l}\text { Apoyo social } \\
\text { confidencial }\end{array}$} & \multicolumn{3}{|c|}{$\begin{array}{c}\text { Apoyo social } \\
\text { afectivo }\end{array}$} & \multicolumn{3}{|c|}{ Apoyo marido } \\
\hline & Promedio & DE & r & Promedio & $\mathrm{DE}$ & r & Promedio & $\mathrm{DE}$ & r & Promedio & DE & r \\
\hline Escolaridad de la madre & & & $-0,45$ & & & 0,14 & & & 0,32 & & & 0,13 \\
\hline Ninguna/primaria incompleta & 12,62 & 7,89 & & 1,17 & 4,93 & & 3,00 & 6,50 & & 3,00 & 7,94 & \\
\hline Primaria/bachiller incompleto & 9,56 & 5,75 & & 0,85 & 4,61 & & 4,56 & 5,18 & & 2,86 & 8,40 & \\
\hline Bachiller/universidad & 4,75 & 5,95 & & 3,21 & 4,98 & & 7,79 & 4,50 & & 6,11 & 8,94 & \\
\hline Índice de posesiones & & & $-0,42$ & & & 0,24 & & & 0,29 & & & 0,21 \\
\hline Bajo (0-1) & 12,06 & 7,34 & & $-0,06$ & 4,83 & & 3,10 & 5,91 & & 1,75 & 8,18 & \\
\hline Medio (2-3) & 9,21 & 6,42 & & 2,32 & 4,69 & & 5,30 & 5,72 & & 3,97 & 8,91 & \\
\hline Alto (4-5) & 3,94 & 6,00 & & 3,10 & 4,88 & & 7,62 & 4,47 & & 7,28 & 7,04 & \\
\hline Índice de dependencia & & & 0,36 & & & $-0,08$ & & & $-0,24$ & & & $-0,17$ \\
\hline Menor de 1 & 6,22 & 5,55 & & 1,74 & 5,02 & & 5,81 & 5,85 & & 5,67 & 7,93 & \\
\hline Entre 1 y 1,99 & 8,44 & 7,34 & & 2,19 & 4,65 & & 6,16 & 5,30 & & 4,04 & 8,65 & \\
\hline 2 y más & 13,03 & 6,99 & & 0,72 & 5,15 & & 2,37 & 5,65 & & 1,80 & 8,56 & \\
\hline Estado civil & & & $-0,012$ & & & $-0,10$ & & & $-0,08$ & & & \\
\hline Casada/unión libre & 9,31 & 6,96 & & 1,98 & 4,79 & & 5,30 & 5,38 & & 6,25 & 7,70 & \\
\hline Separada & 6,96 & 5,93 & & 1,16 & 5,11 & & 4,45 & 7,05 & & $-3,81$ & 9,35 & \\
\hline Soltera/viuda & 10,81 & 9,81 & & 0,35 & 5,31 & & 3,96 & 5,01 & & & & \\
\hline Ingresos & & & $-0,13^{\star}$ & & & 0,25 & & & 0,07 & & & $-0,08$ \\
\hline No tiene ingresos & 9,28 & 6,62 & & 0,75 & 4,96 & & 4,82 & 6,01 & & 4,47 & 8,54 & \\
\hline Menos tercera parte SMM & 11,62 & 8,31 & & 2,23 & 4,08 & & 4,16 & 5,61 & & 2,56 & 7,85 & \\
\hline Entre $1 / 3$ y el SSM & 7,42 & 6,50 & & 2,16 & 5,88 & & 4,74 & 4,93 & & 2,63 & 8,78 & \\
\hline Más de SMM & 3,64 & 6,20 & & 5,71 & 3,50 & & 8,65 & 4,31 & & 4,47 & 9,70 & \\
\hline Salud percibida & & & 0,24 & & & $-0,14$ & & & $-0,11$ & & & $-0,09$ \\
\hline Muy buena/buena & 8,40 & 7,50 & & 2,25 & 4,70 & & 5,48 & 5,66 & & 4,62 & 7,95 & \\
\hline Regular & 10,28 & 6,52 & & 0,84 & 5,18 & & 4,52 & 6,03 & & 2,81 & 9,27 & \\
\hline Mala/muy mala & 13,30 & 6,99 & & 0,40 & 4,72 & & 2,90 & 4,84 & & 2,50 & 9,02 & \\
\hline
\end{tabular}

Correlaciones en negrilla corresponden a $\mathrm{p}<0,05$.

* $\mathrm{p}<0,10$

SMM = salario mínimo mensual, estimado en $\$ 332.000$ pesos (US\$110).

4, 5, 10 y 11, representando el apoyo social afectivo. Mientras que el segundo factor, representado por los ítems 6,7 y 8 resume la dimensión de apoyo confidencial. Los dos factores explicaron el $45,0 \%$ de la varianza total. La consistencia interna de cada factor es alta considerando el alfa de Cronbach de 0,70. Los factores tuvieron una correlación de 0,52. La sumatoria de valores iniciales fue utilizada para crear un resultado de apoyo afectivo y confidencial. Las variables muestran asimetría positiva y varios puntos modales. El promedio de apoyo afectivo fue de 5,05 , siendo la sumatoria de valores positiva para el $80,0 \%$ de las madres, mientras que el apoyo confidencial presenta un promedio de 1,67 , siendo positiva la sumatoria para el 66,0\% de las madres entrevistadas. Los análisis factoriales para el apoyo del esposo/compañero fueron realizados en 171 madres casadas, en unión libre o separadas. Los resultados factoriales mostraron la presencia de un solo factor que explica el $67,0 \%$ de la varianza total (Tabla 5). El 59,0\% de las madres tenían un resultado positivo para el apoyo social del esposo/compañero.

\section{Relación con variables predictoras}

La Tabla 3 muestra las correlaciones parciales entre las variables predictoras y las variables de seguridad alimentaria y apoyo social. Exceptuando los ingresos de la madre, la escolaridad, 
Análisis factorial de la escala de inseguridad alimentaria $(n=193)$.

\begin{tabular}{|c|c|c|c|}
\hline Ítems & Saturación factor & Promedio (DE) & Nunca $(\%)$ \\
\hline \multicolumn{4}{|l|}{ En los últimos 6 meses } \\
\hline Falta dinero para comprar alimentos & 0,54 & $1,13(0.91)$ & 22,8 \\
\hline Disminuye el número usual de comidas por falta de dinero & 0,78 & $1,00(0,83)$ & 25,9 \\
\hline Disminuye el número de comidas adulto por falta de dinero & 0,83 & $0,85(0,81)$ & 35,8 \\
\hline Come menos un adulto en la comida principal & 0,74 & $0,72(0,78)$ & 42,5 \\
\hline Se queja de hambre un adulto por falta de alimentos & 0,86 & $0,68(0,74)$ & 44,6 \\
\hline Se acuesta con hambre un adulto por falta de alimentos & 0,89 & $0,69(0,68)$ & 39,9 \\
\hline Algún miembro come menos de lo que desea por falta de dinero & 0,84 & $0,81(0,75)$ & 33,7 \\
\hline Disminuye en un niño el número de comidas por falta de dinero en el hogar & 0,85 & $0,68(0,70)$ & 43,5 \\
\hline Algún niño come menos en la comida principal por que no alcanzan los alimentos & 0,82 & $0,61(0,72)$ & 49,0 \\
\hline Algún niño se queja de hambre por falta de alimentos & 0,86 & $0,64(0,74)$ & 47,2 \\
\hline Se compran menos alimentos indispensables para niños & 0,74 & $0,90(0,80)$ & 30,1 \\
\hline Algún niño se acuesta con hambre, no alcanza comida & 0,81 & $0,56(0,70)$ & 51,8 \\
\hline Total & & $9,31(7,43)$ & \\
\hline Alfa de Cronbach & & 0,90 & \\
\hline
\end{tabular}

el índice de pertenencias y el índice de dependencia económica se correlacionan de manera negativa con la seguridad alimentaria. Así pues, las madres más escolarizadas y en mejor estado socioeconómico presentan menor resultado en el índice de seguridad alimentaria y, por lo tanto, hogares más seguros. Igualmente, un índice de dependencia mayor se relaciona con mayor inseguridad alimentaria. Mientras que el apoyo social se correlaciona de manera positiva con la escolaridad de la madre y el índice de posesiones materiales, siendo las madres más escolarizadas y de mejor nivel económico las que perciben apoyo social más alto. Las madres casadas o en unión libre presentan mayor apoyo confidencial y afectivo que las madres separadas, y que las madres solteras y viudas, aunque estas diferencias no fueron significativas. Las mujeres con salud percibida muy buena/buena presentan los promedios más bajos de inseguridad alimentaria, y los más altos para las variables de apoyo social (Tabla 3). Las redes sociales se correlacionan de manera positiva con el apoyo social (Tabla 6), de modo que el apoyo del confidente es más positivo entre mayor numero de familiares y contactos visuales se informen, y mayor sea el grado de participación social; mientras que el apoyo afectivo es mayor en las madres que indiquen más numero de amigos y contacto visual con ellos. El apoyo del marido es independiente de las redes familiares y de amigos, y de la integración social.

\section{Relación con el estado nutricional}

El promedio de resultado $\mathrm{Z}$ para nuestra población de estudio se encuentra por debajo del promedio de la población de referencia para los dos indicadores, -0,65 (ED: 1,04) para tallapara-edad (TPE) y de -0,25 (ED: 1,03) para peso-para-talla (PPT), con una prevalencia total de desnutrición crónica de $9,8 \%$ y de desnutrición aguda de 2,6\%. La seguridad alimentaria, el apoyo social afectivo y el apoyo del compañero se relacionan de manera positiva con el estado nutricional, representado tanto en los promedios de $\mathrm{Z}$ resultado de talla-para-edad (TPE) como en la frecuencia de desnutrición (Tabla 7). Cualquier grado de inseguridad alimentaria se asocia con la presencia de desnutrición, igualmente, que un apoyo social afectivo alto se presenta con promedios más elevados en el indicador de TPE, y una proporción significativamente menor de niños en desnutrición. La proporción más baja de desnutrición crónica se encuentran entre aquellos que tienen apoyo confidencial alto $(\mathrm{p}=$ $0,11)$. Los promedios más altos en el indicador de TPE se encuentran en las madres con apoyo social positivo de su compañero, siendo las prevalencias de desnutrición más altas entre aquellas que tienen apoyo bajo y que no tienen compañero. 
Análisis factorial para el apoyo social general (Duke-UNC) y del compañero (OSLCD).

\begin{tabular}{|c|c|c|c|c|}
\hline Items & Factor 1 & Factor 2 & Promedio (DE) & $\begin{array}{c}\text { Valores } \\
\text { negativos (\%) }\end{array}$ \\
\hline \multicolumn{5}{|l|}{ Escala Duke-UNC-11 } \\
\hline 1, Recibo visitas de mis amigos y familiares & * & * & $2,81(2,51)$ & 14,7 \\
\hline 2, Recibo ayuda en asuntos de mi casa** & - & - & $0,50(1,97)$ & 55,2 \\
\hline 3, Recibo elogios y reconocimiento de mi trabajo** & - & - & $1,42(1,77)$ & 31,8 \\
\hline 4, Cuento con personas que se preocupan & 0,75 & & $1,20(2,05)$ & 17,2 \\
\hline 5, Recibo amor y afecto & 0,65 & & $1,34(1,96)$ & 24,5 \\
\hline 6, Posibilidad de hablar de mis problemas en trabajo o casa & * & 0,67 & $0,75(2,04)$ & 16,7 \\
\hline 7, Posibilidad de hablar de mis problemas personales y familiares & * & 0,78 & $0,50(2,10)$ & 27,1 \\
\hline 8, Posibilidad de hablar de mis problemas económicos & * & 0,58 & $0,41(2,05)$ & 31,2 \\
\hline 9, Recibo invitaciones para distraerme y salir con otras personas ${ }^{\star *}$ & - & - & $0,31(2,27)$ & 37,0 \\
\hline 10, Recibo consejos útiles cuando ocurre un acontecimiento importante & 0,63 & * & $1,10(1,88)$ & 38,0 \\
\hline 11, Recibo ayuda cuando estoy enferma en cama & 0,56 & * & $1,41(1,81)$ & 18,8 \\
\hline Alfa de Cronbach & 0,75 & 0,70 & & \\
\hline Promedio & 5,05 & 1,67 & & \\
\hline Desviación Estándar & 5,78 & 4,90 & & \\
\hline Rango & -12 a 12 & -9 a 9 & & \\
\hline \multicolumn{5}{|l|}{ Escala de apoyo del esposo } \\
\hline 1, Esposo ayuda con niños & 0,75 & & $1,64(1,84)$ & 16,7 \\
\hline 2, Esposo ayuda en cosas de la casa & 0,85 & & $1,05(2,19)$ & 16,4 \\
\hline 3, Esposo ayuda cuando está cansada & 0,88 & & $0,54(2,29)$ & 27,5 \\
\hline 4, Esposo ayuda cuando está triste & 0,78 & & $0,38(2,33)$ & 39,2 \\
\hline 5, Esposo ayuda de manera general & 0,83 & & $1,01(2,11)$ & 33,3 \\
\hline Alfa de Cronbach & 0,88 & & & \\
\hline Promedio & 4,64 & & & \\
\hline Desviación Estándar & 8,84 & & & \\
\hline Rango & -15 a 15 & & & \\
\hline
\end{tabular}

* Saturación menor de 0,30.

** Comunidad menor de 0,30.

\section{Discusión}

El índice de Hambre (CCHIP), la encuesta de apoyo social de DUKE-UNC-11, y la encuesta del apoyo de compañero (QLSCD) adaptados en una población de mujeres afro-colombianas de bajo grado de escolaridad y bajos recursos económicos, reproducen las dimensiones de las escalas, presentan validez de constructo en relación con los modelos teóricos propuestos, y tienen alta consistencia interna. Los factores encontrados en nuestros análisis fueron convertidos a variables categóricas que mostraron estar relacionadas con el estado nutricional de los menores de 18 meses: algún grado de inseguridad alimentaria, un bajo apoyo emotivo general y un bajo apoyo del marido se asociaron a menores promedios en el resultado $\mathrm{Z}$ de talla-para-edad, y mayor proporción de desnutrición crónica.

\section{Seguridad alimentaria}

El índice de hambre es una medición cualitativa del grado de inseguridad alimentaría percibido por las madres de la población de estudio. En Colombia y en otros países de Latinoamérica se han aplicado otros instrumentos para medir de manera cualitativa y cuantitativa el grado de inseguridad alimentaria de los hogares 16,17. La medición cuantitativa de la seguridad alimentaria se torna compleja cuando no se tienen estudios previos de la dieta de las poblaciones. Por ello, se enfatiza en la validación de instrumentos simples, de fácil aplicación y de bajo costo 7 . El instrumento utilizado en este estudio muestra fácil aplicación y comprensión en una población de bajo nivel de escolaridad. A diferencia del reporte de Lorenzana \& Sanjur 8, nosotros no encontramos la presencia de dos factores: modificación de la dieta y ex- 
Distribución del apoyo social por niveles de las variables predictoras. Correlaciones parciales no paramétricas.

\begin{tabular}{|c|c|c|c|c|c|c|c|c|c|}
\hline \multirow[t]{2}{*}{ Variables predictoras } & \multicolumn{3}{|c|}{$\begin{array}{l}\text { Apoyo social } \\
\text { confidencial }\end{array}$} & \multicolumn{3}{|c|}{$\begin{array}{c}\text { Apoyo social } \\
\text { afectivo }\end{array}$} & \multicolumn{3}{|c|}{ Apoyo marido } \\
\hline & Media & DE & r & Media & DE & $r$ & Media & DE & r \\
\hline Numero de amigos & & & 0,08 & & & 0,12 & & & 0,09 \\
\hline Primero (0-5) & 0,88 & 4,86 & & 3,70 & 5,62 & & 2,22 & 8,49 & \\
\hline Segundo & 1,37 & 4,92 & & 4,47 & 5,97 & & 3,67 & 7,98 & \\
\hline Tercero & 0,85 & 5,52 & & 4,95 & 6,27 & & 1,40 & 9,26 & \\
\hline Cuarto & 2,27 & 4,92 & & 5,90 & 5,53 & & 5,70 & 8,89 & \\
\hline Quinto & 2,36 & 4,65 & & 5,79 & 5,77 & & 4,62 & 7,92 & \\
\hline Contacto visual amigos & & & 0,07 & & & 0,11 & & & 0,02 \\
\hline Primero (0-5) & 1,32 & 4,58 & & 3,88 & 5,35 & & 4,44 & 8,40 & \\
\hline Segundo & 1,09 & 5,34 & & 4,41 & 6,67 & & 3,25 & 9,02 & \\
\hline Tercero & 3,00 & 3,78 & & 5,29 & 5,92 & & $-0,07$ & 9,59 & \\
\hline Cuarto & 0,83 & 4,91 & & 5,15 & 5,12 & & 4,85 & 7,40 & \\
\hline Quinto & 3,20 & 4,68 & & 6,49 & 5,39 & & 4,41 & 8,66 & \\
\hline Numero de familiares & & & 0,13 & & & 0,04 & & & 0,004 \\
\hline Primero $(0-5)$ & 0,84 & 4,46 & & 3,79 & 5,60 & & 4,68 & 6,45 & \\
\hline Segundo & 0,28 & 5,17 & & 5,21 & 5,93 & & 4,28 & 8,57 & \\
\hline Tercero & 1,68 & 5,59 & & 6,16 & 5,24 & & 1,47 & 10,03 & \\
\hline Cuarto & 2,75 & 5,07 & & 5,38 & 5,73 & & 4,30 & 8,87 & \\
\hline Quinto & 2,68 & 3,78 & & 4,47 & 6,31 & & 4,55 & 8,19 & \\
\hline Contacto visual familiares & & & 0,13 & & & 0,08 & & & $-0,024$ \\
\hline Primero $(0-5)$ & 1,10 & 4,24 & & 4,92 & 5,66 & & 4,92 & 8,37 & \\
\hline Segundo & 0,11 & 4,95 & & 3,57 & 6,14 & & 3,80 & 7,96 & \\
\hline Tercero & 2,27 & 5,34 & & 5,39 & 6,31 & & 3,68 & 9,28 & \\
\hline Cuarto & 0,98 & 4,94 & & 4,25 & 4,82 & & 2,62 & 7,95 & \\
\hline Quinto & 3,68 & 4,45 & & 6,79 & 5,63 & & 4,34 & 9,05 & \\
\hline Integración social & & & 0,17 & & & $-0,08$ & & & 0,016 \\
\hline Alta (4-5) & 3,43 & 3,15 & & 5,43 & 6,45 & & $-0,86$ & 10,09 & \\
\hline Media (2-3) & 2,75 & 4,83 & & 5,50 & 5,99 & & 4,30 & 8,43 & \\
\hline Baja (0-1) & 0,72 & 0,72 & & 4,60 & 5,58 & & 3,85 & 8,44 & \\
\hline
\end{tabular}

Correlaciones en negrilla corresponden a $\mathrm{p}<0,05$.

periencia de hambre. Sin embargo, se encuentra que la severidad de la seguridad alimentaria se relaciona con la sensación de hambre 17 . Por ejemplo, la experiencia de hambre en adultos (Tabla 1, pregunta 6) se presenta en $57,1 \%$ de los hogares severamente inseguros, mientras que está proporción es de $2,9 \%$ en los hogares moderadamente inseguros y de $0,0 \%$ para los seguros. En los niños (Tabla 1, pregunta 12), la distribución es $71,4 \%$ y 2,9\% para los hogares severa y moderadamente inseguros, y $0,0 \%$ para los seguros. Además, la escala en nuestra población muestra validez de constructo con el modelo conceptual de los mismos autores 8: se correlaciona con el estado socioe- conómico, la escolaridad, el índice de dependencia económica y el nivel de ingresos de la madre. Las relaciones entre la seguridad alimentaria y las condiciones socioeconómicas han sido informadas en Estados Unidos 18, en países africanos 19, y más recientemente en Brasil 7. Igualmente, la relación alcanzada en este estudio entre la inseguridad alimentaria y la salud percibida ha sido descrita previamente 20 .

\section{Apoyo social}

El instrumento de DUKE-UNC-11 de nuestro estudio mostró que los ítems 1, 2, 3 no aportan al modelo factorial, y que la solución factorial 
Características de las madres de niños de 6 a 18 meses en Guapi según nivel de inseguridad alimentaria, apoyo social (AS) y su relación con el estado nutricional.

\begin{tabular}{|c|c|c|c|c|c|}
\hline \multirow[t]{2}{*}{ Variables } & \multirow[t]{2}{*}{ Total \% (n) } & \multicolumn{2}{|c|}{ Talla-para-edad } & \multicolumn{2}{|c|}{ Peso-para-talla } \\
\hline & & Promedio (EE) & $\% *$ & Promedio (EE) & $\% \star$ \\
\hline \multicolumn{6}{|l|}{ Seguridad alimentaria } \\
\hline Seguro & $17,1(33)$ & $-0,11(0,18)^{\star \star}$ & $3,0^{\star \star \star}$ & $-0,14(0,18)$ & 0,0 \\
\hline Levemente inseguro & $61,7(119)$ & $-0,79(0,09)$ & 14,3 & $-0,29(0,09)$ & 3,4 \\
\hline Inseguridad moderada & $17,6(34)$ & $-0,67(0,17)$ & 0,0 & $-0,16(0,18)$ & 2,9 \\
\hline Inseguridad severa & $3,6(7)$ & $-0,87(0,37)$ & 14,3 & $-0,66(0,40)$ & 0,0 \\
\hline \multicolumn{6}{|l|}{ AS confidencial } \\
\hline Negativo $(-9$ a -1$)$ & $33,7(65)$ & $-0,67(0,13)$ & 13,8 & $-0,41(0,13)$ & 3,1 \\
\hline Bajo (0-4) & $35,1(68)$ & $-0,67(0,13)$ & 11,8 & $-0,14(0,12)$ & 2,9 \\
\hline Positivo (5-9) & $31,2(60)$ & $-0,60(0,14)$ & 3,3 & $-0,21(0,13)$ & 1,7 \\
\hline \multicolumn{6}{|l|}{ AS afectivo } \\
\hline Negativo $(-12 \mathrm{a}-1)$ & $20,0(38)$ & $-1,09(0,17)^{\star \star}$ & 18,4 & $-0,51(0,17)$ & 0,0 \\
\hline Bajo (0-5) & $24,9(48)$ & $-0,61(0,15)$ & 10,4 & $-0,26(0,15)$ & 6,3 \\
\hline Positivo (6-12) & $55,1(107)$ & $-0,52(0,10)$ & 6,5 & $-0,16(0,10)$ & 1,9 \\
\hline \multicolumn{6}{|l|}{ AS compañero } \\
\hline Negativo $(-15$ a -1$)$ & $25,9(53)$ & $-0,92(0,14) \#$ & $5,7 \#$ & $-0,22(0,14)$ & $5,7^{\star \star \star}$ \\
\hline No tiene & $15,1(22)$ & $-0,75(0,22)$ & 13,6 & $-0,31(0,22)$ & 9,1 \\
\hline Bajo (0-7) & $18,0(40)$ & $-0,61(0,16)$ & 20,0 & $-0,28(0,16)$ & 0,0 \\
\hline Positivo (8-15) & $41,0(78)$ & $-0,46(0,12)$ & 6,4 & $-0,25(0,12)$ & 0,0 \\
\hline
\end{tabular}

* Proporción de niños con < -2 DE.

$\star \star * p<0,01$.

$\# p<0,10$.

de nuestra población de estudio fue muy similar al estudio inicial 9. Dos factores fueron encontrados en el estudio de validación inicial, uno que resume los ítems 6, 7, 8, 9 y 10 dentro del apoyo confidencial, nosotros encontramos los 3 primeros. El apoyo afectivo encontrado en el estudio de Broadhead et al. 9 estaba saturado por las variables 4,5 y 11 , mientras que nosotros encontramos 4, 5, 10 y 11 , siendo la única diferencia que la variable "recibo consejos útiles cuando ocurre algo importante en mi vida" se torna una variable afectiva en nuestro estudio más que una variable de confidente. Un estudio realizado en España entre población marginal encontró que el ítem 10 del cuestionario se relaciona con el apoyo afectivo, igual que nuestro estudio 21. Mientras que Bellon et al. 11 muestra en poblaciones más escolarizadas, que el apoyo afectivo lo reúnen los ítems 3, 4, 5 y 11, y el confidente los ítems 1, 2, 6, 7, 8, 9 y 10. En general, la concordancia de los factores es muy similar entre los estudios anteriores y el nuestro. Las diferencias en la saturación de los factores y la no-inclusión de los ítems 1, 2, 3 y 9 podrían indicar diferentes patrones culturales de afecto, o la inexistencia de las expresiones apoyo 22 . El contexto rural de la población facilita el contacto de las personas y ofrece pocos sitios de entretenimiento, lo cual explicaría que los ítems 1 y 9 que se relacionan con las visitas y las invitaciones no se agrupen dentro de las dimensiones de soporte. Por otro, el ítem 3 se relaciona con los elogios en el trabajo, siendo $61,0 \%$ amas de casa o estudiantes.

Las dos dimensiones de apoyo social se relacionaron con los indicadores socioeconómicos. En este sentido se describe que las personas de baja clase social (medida por el nivel educativo o por los ingresos) perciben apoyo social emotivo más bajo que aquellas de clases sociales superiores 22,23 . Igualmente, las variables se correlacionan de la manera sugerida en el modelo de Berkman et al. 15. Por ejemplo, el mayor contacto con familiares predice mayor apoyo confidencial, mientras que el apoyo emotivo está relacionado con él numero de amigos y la frecuencia del contacto visual con ellos. Asimismo, un bajo grado de apoyo social 
se relacionó con una peor percepción de la salud, como lo soportan otros estudios 24 .

La encuesta de apoyo del padre del niño (QSLCD) muestra la presencia de un solo factor que agrupa el apoyo instrumental, afectivo y confidencial. Hasta la fecha no hay ningún informe publicado con el cual comparar los resultados encontrados en el análisis factorial. La escala tiene alta consistencia interna y validez de constructo con el modelo de Teoría de Redes Sociales propuesto por Berkman et al. 15. El apoyo se relacionó con el nivel educativo y el índice de posesiones; las madres casadas o en unión libre presentaron promedios más altos que las separadas; y cuanto mayor era la dependencia familiar, menor era el soporte percibido.

\section{Seguridad alimentaria, apoyo} y estado nutricional

Nuestros resultados preliminares en una población con proporción de malnutrición crónica y aguda bajas ( $9,8 \%$ y $2,3 \%$ respectivamente) sugieren la relación entre la seguridad alimentaria y el apoyo social con el estado nutricional infantil. Recientes reportes en países desarrollados 25,26 y en países en desarrollo 4,27 sugieren que la inseguridad alimentaria afecta la salud infantil, y en nuestro caso el estado nutricional en el indicador de talla-para-edad. Los mecanismos implicados en la relación entre la seguridad alimentaria y el estado nutricional pueden explicarse por la disminución de la ingesta de alimentos, disminución en la calidad y diversidad de la dieta, y deficiencias de micronutrientes 27,28; o por el aumento de la frecuencia de otros estados de salud, infecciones, que a su vez afectan el estado nutricional 18,29. Por otro lado, los trabajos reportados por Zeitlin et al. 2,30 sugieren, como el modelo del Redes Sociales 15 , que los efectos del apoyo social sobre el estado nutricional infantil pueden ser mediados por su influencia en las prácticas de cuidado, en los estados de salud la madre, en la auto-estima, la competencia social, o estados de depresión. Aunque los mecanismos a través de los cuales el apoyo social puede ejercer su influencia sobre el estado nutricional no han sido elucidados, estudios recientes muestran algunas relaciones. Citamos, como ejemplos, el efecto del estado mental de la madre sobre el estado nutricional infantil 31 , o el efecto del apoyo del padre sobre las prácticas de lactancia y prevención 32 . El apoyo social también podría afectar el estado nutricional del niño a través de mecanismo directos (Ej. fisiológicos relacionados con respuestas al estrés). Así se describe que las madres con escaso apoyo tienden a tener niños con más bajo peso al nacer y partos pre-término 33 .

\section{Recomendaciones}

La adecuada medición de los recursos para el cuidado constituye un reto fundamental en la comprensión de las causas de la malnutrición y en la implementación de programas de intervención 1. La medición de la seguridad alimentaria mediante instrumentos simples y validados puede servir como alternativa de diagnóstico del estado nutricional de la población y como medidor de la calidad de la dieta 34,35 .

En este estudio presentamos la validación de tres instrumentos que pueden ser utilizados en poblaciones de bajo nivel de escolaridad, fácilmente adaptables a las condiciones particulares de cada comunidad y fácilmente aplicados por proveedores de salud primarios o por profesionales de la salud. Algunos pasos adicionales para la implementación de estos instrumentos estarían relacionados con la replicabilidad, la validación concurrente contra un "estándar de oro" de adecuación de la dieta en el caso de la escala de inseguridad alimentaria, y la medición de la consistencia de los hallazgos en otros contextos.

En cuanto a las asociaciones encontradas entre las dimensiones de seguridad alimentaria y apoyo social con el estado nutricional infantil, se sugieren futuras preguntas de investigación: ¿Cuál es el papel de las prácticas de cuidado-lactancia, alimentación complementaria y prevención-en las relaciones entre la seguridad alimentaria y el apoyo social, con el estado nutricional infantil? ¿La relación entre el apoyo social y estado nutricional después de los 6 meses de edad, podría estar mediada por mecanismos prenatales como el peso al nacer? 


\section{Resumen}

Nosotros realizamos un estudio transversal en 193 madres de niños de 6 a 18 meses de edad de una comunidad afro-colombiana con los objetivos: (1) adaptar y validar la escala de hambre del Community Childhood Hunger Identification Project, de apoyo social de DUKE-UNC-11, y de apoyo del compañero del Québec Longitudinal Study of Child Development (QLSCD), $e$ (2) identificar la relación entre la inseguridad alimentaria y el soporte social con el estado nutricional infantil. Se midió la validez de constructo mediante análisis factoriales y correlaciones no-paramétricas basadas en modelos teóricos. Se establecieron los resultado-Z de talla-para-edad y peso-para-talla. El análisis factorial redujo la escala de hambre y la escala de QLSCD a un factor; y la escala de DUKE-UNC11 a dos factores. El alfa de Cronbach osciló entre 0,70 y 0,90. Las tres escalas se correlacionaron con las condiciones sociales. Las escalas de apoyo se correlacionaron con las redes sociales y la salud percibida de la madre. La inseguridad alimentaria y el apoyo social negativo (emocional y del compañero) se asociaron a menores índices talla-para-edad, y mayor proporción de desnutrición crónica. El estudio apoya la utilidad de los instrumentos presentados en la medición de los conceptos planteados.

Apoyo Social; Estado Nutricional; Bienestar del Niño

\section{Colaboradores}

Todos los autores participaron en la elaboración del protocolo. La investigación de campo y los análisis fueron realizados por B. E. Alvarado. El manuscrito fue elaborado por B. E. Alvarado y revisado por los demás autores.

\section{Agradecimientos}

Los autores agradecen de manera especial a las madres y niños de Guapi que participaron en este estudio. Además a Adela, Luisa, Carmen, Nidia y Stella que colaboraron en la recolección de los datos. Al Dr. Julio César Reina por el entrenamiento en mediciones antropométricas y al Dr. Jairo Osorno por sus valiosos aportes al protocolo. Este proyecto ha sido financiado por la Organización Panamericana de la Salud - Programa de subvenciones de Tesis de Salud Pública. Referencia: HDP/HDR/RG-T/COL/3146 y por Colciencias, Convocatoria 2001, Plan Nacional de Ciencia y Tecnología de la Salud (código del proyecto: 1103-04-11985). El proyecto ha contado con la colaboración del personal docente y administrativo del Département de Medicine Sociale et Santé Préventive de la Université de Montréal.

\section{Referencias}

1. Engle PL, Bentley M, Pelto G. The role of care in nutrition programmes: current research and a research agenda. Proc Nutr Soc 2000; 59:25-35.

2. Zeitlin M, Ghassemi H, Mansour M. Positive deviance in child nutrition: with emphasis on psychological and behavioral aspects and implications for development. Tokyo: United Nations University Press; 1990.

3. Zeitlin M. Child care and nutrition: the finding from positive deviance research. Ithaca: Cornell International Nutrition; 1996. (Monograph Series).

4. Begin F, Frongillo Jr. EA, Delisle H. Caregiver behaviors and resources influence child height-forage in rural Chad. J Nutr 1999; 129:680-6.

5. Carvalhaes MA, Benicio MH. Capacidade materna de cuidar e desnutrição infantil. Rev Saúde Pública 2002; 36:188-97.

6. Rahman A, Lovel H, Bunn J, Iqbal Z, Harrington R. Mothers' mental health and infant growth: a case-control study from Rawalpindi, Pakistan. Child Care Health Dev 2004; 30:21-7.

7. Departamento de Medicina Preventiva e Social; Faculdade de Ciências Médicas; Universidade Estadual de Campinas. Acompanhamento e avaliação da seguranca alimentar das familias brasileiras: validação de metodologia e de instrumento de coleta de informação urbano/rural. Campinas: Departamento de Medicina Preventiva e Social, Faculdade de Ciências Médicas, Universidade Estadual de Campinas; 2004. (Relatorio Técnico).

8. Lorenzana PA, Sanjur D. Abbreviated measures of food sufficiency validly estimate the food security level of poor households: measuring household food security. J Nutr 1999; 129:687-92.

9. Broadhead WE, Gehlbach SH, De Gruy FV, Kaplan BH. The Duke-UNC functional social support questionnaire. Measurement of social support in family medicine patients. Med Care 1988; 26:70923.

10. Institut de la Statistique de Quebec. Longitudinal study of child development in Quebec. Quebec: Institut de la Statistique de Quebec; 2000.

11. Bellon-Saameno JA, Delgado-Sanchez A, Lunadel-Castillo JD, Lardelli-Claret P. Validez y fiabilidad del cuestionario de apoyo social funcional Duke-UNC-11. Aten Primaria 1996; 18:153-63.

12. Zaldivar F, Rubio VJ, Morales JM, Zunzunegui MV. Factores de riesgo de maltrato infantil: un estudio de casos y controles en el área metropolitana de Madrid. Revista de Psicología de la Salud 1998; 10:53-77.

13. Tabares E, Alvarado BE. Estado nutricional y prácticas alimentarias en los primeros 18 meses de vida en poblaciones Amerindias y Afro-Colombianas de la Costa Pacífica. AntroPacífico 2003; 1:1726.

14. Profamilia. Encuesta nacional de demografia y salud. Santa Fé de Bogotá: Profamilia; 2000.

15. Berkman LF, Glass T, Brissette I, Seeman TE. From social integration to health: Durkheim in the new millennium. Soc Sci Med 2000; 51:843-57.

16. Lareo LR, Gracia BN, Fajardo L, Romero LH, Ac- 
ciarri G, Pradilla A, et al. From food basket to food security. The food factor in nutritional surveillance. Arch Latinoam Nutr 1990; 40:22-43.

17. Frongillo Jr. EA. Validation of measures of food insecurity and hunger. J Nutr 1999; 129 (2S Suppl):506S-9S.

18. Rose D. Economic determinants and dietary consequences of food insecurity in the United States. J Nutr 1999; 129 (2S Suppl):517S-20S.

19. Garrett JL, Ruel M. Are determinants of rural and urban food security and nutritional status different? Some insights from Mozambique. World Development 1999; 27:1955-75.

20. Vozoris NT, Tarasuk VS. Household food insufficiency is associated with poorer health. J Nutr 2003; 133:120-6.

21. De la Revilla L, Bailon E, Luna JD, Delgado A, Prados MA, Fleitas L. Validación de una escala de apoyo social funcional para uso en la consulta del médico de familia. Aten Primaria 1991; 8:688-92.

22. Turner RJ, Marino F. Social support and social structure: a descriptive epidemiology. J Health Soc Behav 1994; 35:193-212.

23. Mickelson KD, Kubzansky LD. Social distribution of social support: the mediating role of life events. Am J Community Psychol 2003; 32:265-81.

24. Steptoe A, Marmot M. Burden of psychosocial adversity and vulnerability in middle age: associations with biobehavioral risk factors and quality of life. Psychosom Med 2003; 65:1029-37.

25. Olson CM. Symposium: advances in measuring food insecurity and hunger in the U.S. Introduction. J Nutr 1999; 129 (2S Suppl):504S-5S.

26. Olson CM. Nutrition and health outcomes associated with food insecurity and hunger. J Nutr 1999; 129 (2S Suppl):521S-4S.

27. Tarini A, Bakari S, Delisle H. The overall nutritional quality of the diet is reflected in the growth of Nigerian children. Sante 1999; 9:23-31.
28. Delisle H. La securite alimentaire, ses liens avec la nutrition et la sante. Rev Can Etud Dev 1998; 23:307-29.

29. Allen LH. Nutritional influences on linear growth: a general review. Eur J Clin Nutr 1994; 48 Suppl 1:S75-89.

30. Zeitlin MF, Ahmed NU. Nutritional correlates of frequency and length of breastfeeds in rural Bangladesh. Early Hum Dev 1995; 41:97-110.

31. Baker-Henningham $\mathrm{H}$, Powell $\mathrm{C}$, Walker $\mathrm{S}$, Grantham-McGregor S. Mothers of undernourished Jamaican children have poorer psychosocial functioning and this is associated with stimulation provided in the home. Eur J Clin Nutr 2003; 57:786-92.

32. Brugha R, Kevany J. Determinants of nutrition status among children in the eastern region of Ghana. J Trop Pediatr 1994; 40:307-11.

33. Feldman PJ, Dunkel-Schetter C, Sandman CA, Wadhwa PD. Maternal social support predicts birth weight and fetal growth in human pregnancy. Psychosom Med 2000; 62:715-25.

34. Studdert LJ, Frongillo Jr. EA, Valois P. Household food insecurity was prevalent in Java during Indonesia's economic crisis. J Nutr 2001; 131:268591.

35. Perez-Escamilla R, Ferris AM, Drake L, Haldeman L, Peranick J, Campbell M, et al. Food stamps are associated with food security and dietary intake of inner-city preschoolers from Hartford, Connecticut. J Nutr 2000; 130:2711-7.

Recibido el 24/May/2004

Versión final presentada el 29/Sep/2004

Aprobado el 26/Oct/2004 